Itinéraires Itinéraires

Littérature, textes, cultures

\title{
De Hiroshima aux Twin Towers : les désignants d'événements, une mémoire de l'actualité ?
}

Laura Calabrese Steimberg

\section{OpenEdition}

Journals

Édition électronique

URL : http://journals.openedition.org/itineraires/157

DOI : 10.4000/itineraires.157

ISSN : 2427-920X

Éditeur

Pléiade

\section{Édition imprimée}

Date de publication : 1 juillet 2011

Pagination : 113-127

ISBN : 978-2-296-54673-8

ISSN : 2100-1340

Référence électronique

Laura Calabrese Steimberg, "De Hiroshima aux Twin Towers : les désignants d'événements, une mémoire de l'actualité ? », Itinéraires [En ligne], 2011-2 | 2011, mis en ligne le 03 février 2015, consulté le 20 avril 2019. URL : http://journals.openedition.org/itineraires/157 ; DOI : 10.4000/itineraires.157

\section{(C) $(\oplus \Theta$}

Itinéraires est mis à disposition selon les termes de la licence Creative Commons Attribution - Pas d'Utilisation Commerciale - Pas de Modification 4.0 International. 


\title{
De Hiroshima aux Twin Towers : les désignants d'événements, une mémoire de l'actualité?
}

\begin{abstract}
This paper analyzes the ways in which event names in the media help to build historic memory. We will observe the behavior of media event-names in the press (The swine $f l u, 9 / 11)$, which help us to identify and trace events synthetically. From a discourse perspective, we raise the question of the marks at the surface of the text that allow us to interpret those expressions as events. Our hypothesis is that they first arise as noun phrases including an event noun (i.e. the 9/11 terror attacks), but the latter is gradually erased, as media discourse tends to produce synthetic expressions. We would like to show how those expressions organize collective memory, by stocking a great amount of information, by creating periods (post 9/11), comparisons by antonomasia (the Italian 9/11) or series of events (From Hiroshima to 9/11). Those examples show that event names organize historic memory by marking the rhythm of social life.
\end{abstract}

Keywords : event names, discursive memory, media discourse, naming, media event Mots clés : désignants d'événements, mémoire discursive, discours médiatique, nomination, événement médiatique

\section{Introduction}

Nommer des événements est l'une des principales fonctions du discours d'information médiatique. Le produit de cet acte de dénomination, que nous appelons "désignants d'événements » (cf. infra), a une portée pragmatique qui n'échappe à aucun lecteur de la presse quotidienne et qu'il convient de décrire de manière systématique, dans le cadre d'une sémantique des discours sociaux. Les désignants d'événements (désormais $\mathrm{DE}$ ) ont pour tâche de classer l'événement et le rendre retraçable dans le discours d'information, ainsi que dans leur passage vers d'autres genres de discours (scolaire, politique, conversation quotidienne). En ce sens, ils ont un rôle mémoriel évident, qui ressort dans les exemples suivants : 
(1) Être marocain en Espagne après le « $11-M$ » (Lenouvelobs.com, 9 avril 2004)

(2) Banlieues : les médias américains sans complaisance (Lemonde.fr, 14 novembre 2005)

(3) L'infernal retour de Tchernobyl (Politis, 12 décembre 2002)

(4) Au Guatemala sinistré, l'ouragan Stan a emporté routes et ponts (Lemonde.fr, 19 octobre 05)

Nous pouvons dire, à la suite de Sophie Moirand (2004), que les DE fonctionnent comme des « déclencheurs mémoriels », ce qui signifie que leur mention suffit à évoquer les coordonnées de l'événement (qui, où, quoi), et constituent par là un support de la mémoire de l'actualité.

Il ressort cependant des exemples cités que tous les DE n'ont pas la même capacité d'évocation; ainsi, le rappel que Stan est le nom qui a été donné à un ouragan est nécessaire pour faire référence à un événement, tandis que le 11-M ou Tchernobyl suffisent à actualiser le sens événementiel du désignant. Cependant, Tchernobyl peut circuler bien après les faits, tandis que le 11-M sera adapté au contexte de communication : il deviendra les attentats du 11 mars ou bien les attentats de Madrid dans le discours d'information francophone, tandis qu'en Espagne il continuera à circuler sous sa forme abrégée, el 11-M. Quant à banlieues, notre mémoire de l'actualité (géographiquement ancrée) nous permet de l'actualiser en fonction événementielle et non comme un simple toponyme, quoique pas de façon permanente comme c'est le cas pour Tchernobyl.

Pour expliquer la portée mémorielle des DE et leur fonction d'organisateurs de la matière événementielle, il faut procéder à une description linguistique et discursive, de façon à mettre en évidence les règles de formation qui donnent lieu à ces expressions, autrement dit, pour retracer le processus qui va de la catastrophe de Tchernobyl à Tchernobyl. La description linguistique est ici un préalable à la description en discours. D'une part, elle permet de considérer la base lexicale sur laquelle reposent les expressions servant à désigner des événements (ouragan, catastrophe, guerre). D'autre part, le fait de classer les différentes formes qu'adoptent les $\mathrm{DE}$ en une série de catégories grammaticales permet d'expliquer les usages différenciés que la société en général, et le discours d'information en particulier (mais également le discours historique) réservent aux noms propres et aux dates.

Nous commencerons par décrire les caractéristiques particulières de l'événement médiatique, entité du monde mais dont la condition de possibilité est la mise en discours; ensuite nous aborderons les règles de formation des DE, en rapport avec des pratiques d'écriture et de lecture propres aux sociétés de masse, pour finalement expliquer le rôle qu'ils jouent dans la mise en place d'une mémoire collective de l'histoire immédiate. 


\section{L'événement dans le discours d'information}

L'événement médiatique n'est pas un objet discret, un « realia » homogène et directement disponible à la perception. Bien au contraire, il est un ensemble de faits mis en série par des protocoles sociaux. Nous dirons avec Searle (1998) qu'il s'agit d'un fait institutionnel, dans le sens où il dépend des institutions humaines, et notamment du langage, pour exister ${ }^{1}$. Pour que les entités non physiques (en l'occurrence, institutionnelles) acquièrent une « ontologie objective », selon Searle, elles doivent traverser un processus social de mise en forme. Dans le cas d'un événement médiatique, il s'agit de la construction de l'entité par l'acte de nomination, car « seul le langage permet de désigner des entités dénuées de tout support perceptible direct et de coordonner à distance les individus en communiquant sur des choses qui sont détachées du "ici et maintenant" » (Kaufmann 2008 : 88). C'est pourquoi l'activité de nomination est fondamentale pour donner un statut objectif à l'événement, ainsi que pour le retracer au long de ses transformations et son devenir.

Pour Searle, la nomination d'un fait institutionnel implique l'attribution d'une fonction-statut visant à agir dans l'espace public (un attentat ou une catastrophe exigent des mesures particulières). Or, il est important de constater que cette attribution se fait selon des protocoles d'interprétation qui suivent un patron collectif; autrement dit, la nomination est une activité qui engage tout le corps social et non uniquement les journalistes ${ }^{2}$, comme nous essayons de le montrer dans nos travaux sur la nomination des événements dans la presse (Calabrese 2010). L'attribution de fonction est cruciale pour expliquer la façon dont un événement médiatique se construit et s'installe dans l'espace public, car elle permet de comprendre la nature discursive de l'événement.

D'autres théoriciens soulignent également la dimension langagière comme constitutive de l'événement, que ce soit en philosophie (Badiou 1988), en histoire (Veyne 1996) ou dans des études en communication (Quéré 1994). Ainsi pour Alain Badiou, le nom est ce qui fait point d'arrêt à la dispersion de l'événement, lequel risque de se diluer dans le dénombrement des éléments qui le composent. L'historien Paul Veyne, pour sa part, considère que l'événement n'a pas d'unité réelle mais est le fruit d'un découpage du réel et d'un agencement discursif. Il suggère par ailleurs que le nom donné à l'événement participe activement à cette

1. L'événement historico-médiatique a toujours des conséquences dans le monde extralinguistique (il institue un avant et un après) mais n'est saisissable qu'en discours. Pour qu'une occurrence soit médiatisée, elle a besoin d'être énoncée comme un événement d'intérêt public par un énonciateur autorisé. En dehors de sa médiatisation, il y aura sûrement des victimes, mais pas d'événement médiatique, pas de changement dans l'histoire immédiate. 2. Paradoxalement, c'est le caractère supposé consensuel de l'acte de nomination qui provoque des conflits dans l'espace public, dans la mesure où certaines parties concernées peuvent ne pas se sentir représentées par la dénomination choisie. C'est le cas de mots qui ont une grande charge pragmatique du point de vue du droit international, comme l'emploi du mot génocide pour des événements tels que la crise au Darfour. 
construction. Dans le cadre de ses recherches sur la construction sociale de l'événement, Louis Quéré avance également la thèse de «l'événement sous une description », selon laquelle l'événement ne préexiste pas à sa catégorisation sociale. Par ailleurs, la plupart des citoyens vivant dans une société de masse - ainsi que la plupart des journalistes - ont une connaissance indirecte des événements relayés par le discours d'information. L'acte de nomination fait non seulement exister l'événement, mais il le catégorise en suivant des règles sémantiques implicites dans une société donnée.

Une analyse des discours sociaux soucieuse des matérialités linguistiques doit pour autant se demander comment se construit le sens social de ces entités non discrètes ni immédiatement disponibles à la perception, qui jouent un rôle considérable dans notre perception de l'espace public et dans la construction d'une mémoire commune.

\section{Comment la presse nomme-t-elle les événements?}

La dimension discursive de l'événement, notamment médiatique, se vérifie quotidiennement dans le discours d'information. Ce constat n'implique nullement qu'il n'y a pas de composante factuelle (quelque chose arrive, quelque part, qui modifie le visage de l'espace public), mais que l'acte de nomination fait exister l'événement, le catégorise, lui permet de circuler et est une condition de sa mémorisation par les lecteurs. Pour l'illustrer, prenons l'exemple d'un épisode qui a eu lieu dans l'entourage du président Nicolas Sarkozy. Entre le 7 et le 9 octobre 2009, nous avons pu lire les titres de presse suivants:

(5) L'irrésistible ascension de Jean Sarkozy dans les Hauts-de-Seine (lemonde.fr, 9 octobre 2009)

(6) La gauche s'insurge contre l'ascension fulgurante de Jean Sarkozy (lexpress.fr, 9 octobre 2009)

(7) Jean Sarkozy va succéder à Patrick Devedjian à la tête de l'Établissement public d'aménagement de La Défense, selon lepoint.fr (lenouvelobs.com, 7 octobre 2009)

(8) La Défense : comment Jean Sarkozy a mis Devedjian dehors (marianne2.fr, 8 octobre 2009)

(9) Jean Sarkozy va être élu à la tête de l'établissement de La Défense (ouest-france.fr, 7 octobre 2009)

Quelques jours après, la presse parle de « l'affaire Sarkozy », nous proposant une dénomination pour chapeauter une série de sousévénements perçus comme faisant partie d'un tout :

(10) Affaire Jean Sarkozy: Yade se démarque de l'UMP (nouvelobs.com, 15 octobre 2009) 
(11) L'affaire Jean Sarkozy trouble l'UMP et indigne la gauche (lemonde.fr 14, octobre 2009)

(12) Affaire Jean Sarkozy: les médias aiment trop les fils de (marianne2.fr, 15 octobre 2009)

Nous constatons qu'au niveau de la mise en mots de l'événement, il se produit un fort effet de condensation : les phrases verbales et les désignants d'acteurs (Sarkozy, la gauche, Devedjian) sont subsumées sous une phrase nominale, tandis que l'article défini confère au groupe nominal (l'affaire Jean Sarkozy) le prédicat de réalité des entités objectives. En effet, le syntagme désigne une entité existante et, qui plus est, connue des lecteurs. Dans le cadre du discours d'information, cela implique un partage de la référence à l'échelle sociale. Une fois l'événement « baptisé », le désignant peut remplir son rôle de déclencheur mémoriel :

(13) Élysée : coup de pouce à Pierre Sarkozy? (lefigaro.fr, 8 novembre 2009) Après l'affaire Jean Sarkozy, voici la polémique Pierre Sarkozy. Le site Electronlibre.info affirme que le fils du président, jeune producteur de rap, se serait plaint auprès de l'Élysée alors que la Société civile des producteurs phonographiques (SCPP) lui aurait refusé une aide.

Le désignant est ainsi la pointe de l'iceberg qui englobe les sousévénements qui le composent, mais également les images et les représentations que nous en avons. Dans ce cas précis, par exemple, les représentations qui se dégagent d'un nom propre de notoriété publique font également partie de l'événement même, puisqu'il convoque une série de prédiscours chez le lecteur. Marie-Anne Paveau subsume sous ce concept des repères collectifs tels que « la doxa », « le collectif», « le commun », « le partage » ou « la connaissance encyclopédique » (2006 : 28-33). Les prédiscours constituent « des données antérieures à la mise en langage, d'ordre perceptif et représentationnel, mais cependant préconfigurées par la dimension linguistique »(14). Ils consistent en une série de savoirs, croyances et pratiques «qui donnent des instructions pour la production et l'interprétation du sens en discours » (118), et prédéterminent par là la production de discours dans une société. Ainsi, dans le cas où un lecteur ne serait pas exactement au courant des événements liés à l'affaire Jean Sarkozy, le désignant lui apporte une série d'informations qu'il peut relier à ses connaissances du monde : il s'agit d'un scandale (affaire) à connotation politique qui a lieu dans l'entourage de la majorité (Jean Sarkozy), dont le protagoniste est un fils à papa / un jeune et prometteur politicien de l'UMP (représentations liées à Jean Sarkozy).

Dans le discours d'information générale, les événements sont donc nommés par le biais de noms ou d'expressions nominales, autrement dit, ils sont perçus comme des «substances » dotées de caractéristiques propres. Si la remarque paraît futile, il n'est cependant pas inutile de rappeler la nécessité qu'a le discours médiatique de catégoriser, classer, donner un 
statut aux événements, et donc d'appeler les événements à l'aide d'un nom commun. À la suite des sémanticiens du lexique ${ }^{3}$, nous les appelons « noms événementiels » (désormais Ndév), car ils font référence en langue à des événements et sont compatibles avec des verbes d'action (avoir lieu, survenir). Si la référence des DE dépasse largement le contenu notionnel du nom (comme on l'a vu pour l'affaire Jean Sarkozy, qui évoque des représentations qui dépassent le sens du nom propre et du nom commun), ce contenu notionnel sera un élément important dans notre perception de l'événement.

Pour expliquer la capacité cognitive des DE il est donc nécessaire de passer par une analyse linguistique, dans le but de montrer sur quoi s'appuie le sens et la référence de ces expressions, et comment celles-ci conservent, entretiennent et organisent la mémoire de l'actualité dans une société. Dans le but d'expliquer les rapports entre nomination et mémoire, nous allons observer comment fonctionne la nomination d'événements dans le discours d'information, en proposant un modèle applicable à tous les désignants d'événements.

\section{Les désignants d'événements}

Nous avons choisi l'étiquette "désignants d'événements » pour éviter des imprécisions dans la définition de l'objet. Les expressions que nous rencontrons quotidiennement dans la presse d'information générale peuvent être des noms propres (Tiananmen) ou communs (l'intifada, la canicule), des expressions complètes (la guerre du Golfe) ou incomplètes (la crise); elles peuvent rester longtemps dans la mémoire (les caricatures de Mahomet) ou sortir rapidement du discours public (la catastrophe de Roissy). On peut donc difficilement parler de " noms propres d'événements » pour faire référence à toutes ces expressions, car cela dépend de leur degré de figement et de leur mode de référence : si, comme on vient de le dire, le contenu notionnel joue un rôle dans la référence, celle-ci ne se fait pas de façon directe comme dans le cas du nom propre (voir Calabrese 2009). On peut parler de nom propre dans le cas des séquences figées et des noms qui trouvent une place privilégiée dans la mémoire collective, et vraisemblablement dans les dictionnaires historiques ${ }^{4}$.

Quant à « dénominations d'événements », l'étiquette nous confronte à un autre problème qui touche aux routines rédactionnelles de la presse. En effet, peut-on parler de « dénomination » pour Tiananmen ou le 11 mars, alors que la convention de dénomination ne porte pas sur Tchernobyl mais sur La catastrophe de Tchernobyl (? Cet événement s'appelle Tchernobyl). Ayant réservé l'étiquette de " nom d'événement » aux substantifs à contenu événementiel, et considérant l'ambiguïté qui existe en français entre

3. Par exemple Fabre et Le Draoulec (2006).

4. Voir également Veniard (2007:369-375), qui analyse des expressions servant à désigner des événements en fonction de leur degré de figement. 
nom-noun et nom-name, nous choisissons l'étiquette générique « désignants d'événements ».

\subsection{Les expressions nominales définies incomplètes}

Nous avons donc dit que le discours d'information s'appuie fortement sur des noms événementiels, auxquels viennent s'ajouter des compléments directs (l'affaire Parmalat) ou indirects (la catastrophe de Tchernobyl) pour restreindre la portée du nom. Or, lorsqu'un événement est suffisamment saillant pour une société, une expression incomplète suffit pour opérer la référence, comme dans ces exemples :

(14) La crise, en parler ou pas (lemonde.fr, 15 février 2009)

(15) Les crises irakiennes et palestiniennes avivent la haine des États-Unis (Le Monde, juillet-août 2004)

(16) La canicule, un an après : il faut plus de solidarité (lemonde.fr, 16 juillet 2004)

(17) Météo France prépare un système d'alerte pour prévenir de l'arrivée de la canicule (lemonde.fr, 30 avril 04)

Ces expressions posent le problème de la reconnaissance du référent par l'interlocuteur, et en conséquence de la construction du contexte par la situation d'énonciation. Fortement ancrées dans le contexte, elles n'ont pas besoin de spécifier les coordonnées de l'événement en question. À la question quelle crise? (ex. 14), on pourrait répondre celle-ci, la crise actuelle, la crise financière, etc. Au contraire, l'exemple 15 montre la nécessité de spécifier la nature du nom, autrement dit de le caractériser.

Selon le contexte, le DE peut se présenter sous une forme complète ou incomplète; s'il a souvent besoin de spécifier ses coordonnées, dans d'autres cas un seul groupe nominal suffit à la référenciation. Mais ce dernier cas n'est possible que pendant le moment discursif correspondant, en dehors duquel l'ancrage risque de se perdre, et doit être remplacé par une détermination complète assurant la référenciation. En effet, plus un Ndév est caractérisé, plus il est à même d'assurer la référenciation. L'absence de caractérisation des expressions définies incomplètes produit ainsi un « effet de connivence » (Charolles $2002: 200$ ), qui témoigne de la saillance de l'entité nommée, fût-ce une entité abstraite, comme dans le cas d'un événement. Les éléments nécessaires à l'actualisation des désignants sont disponibles dans les discours qui circulent dans l'espace public, car la localisation de la référence repose sur l'interdiscours médiatique, c'est-à-dire sur les mentions antérieures de la canicule ou de la crise.

Si les noms aptes à intégrer ce type de construction n'abondent pas (crise, canicule, éventuellement guerre), leur fonctionnement est particulièrement éclairant en ce qui concerne leur capacité à conserver la mémoire. En 
effet, les expressions définies incomplètes ont une faible capacité mémorielle et sont souvent complétées par un caractérisant, comme dans le cas de la canicule de $2003^{5}$ (dira-t-on, par ailleurs, à l'avenir la crise de 2008?).

\subsection{Les expressions nominales définies complètes}

Les descriptions définies complètes « comportent des indications sur le contexte dans lequel le critère d'unicité propre au défini est destiné à s'appliquer » (Charolles 2002 : 85). Mais il serait incorrect de penser que leur seul contenu sémantique suffit à opérer la référence, car elles ont une fonction " de renvoi » à l'extérieur du texte, donc un mode de fonctionnement interdiscursif, comme les expressions définies incomplètes. Par exemple, le DE l'affaire Jean Sarkozy, analysé supra, fait référence, sur le site du Nouvel Observateur (puisque la continuité s'établit entre les numéros d'un même journal), à cet énoncé antérieur :

(18) Jean Sarkozy va succéder à Patrick Devedjian à la tête de l'Établissement public d'aménagement de La Défense, selon lepoint.fr (7 octobre 2009)

Mais également à ceux-ci (entre autres), qui ont également contribué à la construction du DE :

(19) Jean Sarkozy, Bachelot, Aubry... les « fils et filles de » en politique (12 octobre 2009)

(20) Jean Sarkozy à l'Epad : «pas lieu d'en faire une polémique » (13 octobre 2009)

(21) Jean Sarkozy à l'Epad : le PS s'élève unanimement (13 octobre 2009)

Les descriptions définies complètes s'inscrivent donc dans un fonctionnement mémoriel typique du discours médiatique. Mais ce qui fait leur particularité est leur tendance à la condensation. Car si le Ndév est fondamental pour catégoriser l'événement et le mémoriser, les données qui l'identifient se trouvent dans le complément du nom : un toponyme, une date, un nom commun (l'affaire du voile, la crise des banlieues) ou un anthroponyme (Jean Sarkozy). Par exemple, dans les affaires (nom événementiel passe-partout au sens peu défini ${ }^{6}$ ) l'anthroponyme est plus signifiant que le nom commun, comme on l'a montré plus haut pour l'affaire Jean Sarkozy.

5. «Sujet de sa dernière pièce présentée chez nous [en Belgique] cet automne : la canicule de l'été 2003. Laurent Ruquier se permettait-il de rire de tout? [de la canicule et de ses conséquences tragiques] » (Zone/02, novembre 2005).

6. Pour M. Lecolle (2009), affaire est un « mot-joker » qui renvoie à des événements de notoriété publique, sans autre spécification. M. Veniard note par ailleurs que, à côté des mots question et scandale, le mot affaire montre « les limites d'une conception exclusivement descriptive du sens. Intuitivement, on ressent ces mots comme caractéristiques des événements dans la presse, bien qu'ils ne décrivent pas l'événement lui-même» (2007 : 457). 
Également, dans la catastrophe de Tchernobyl, Tchernobyl a une force signifiante particulière car elle spécifie la portée du nom ${ }^{7}$. Autrement dit, puisque le caractérisant permet de distinguer l'événement générique du particulier (une affaire quelconque de l'affaire Jean Sarkozy, une catastrophe quelconque de la catastrophe de Tchernobyl), il est d'une certaine façon la partie la plus informative de l'expression.

Dans l'écriture journalistique, les Ndév sont ainsi souvent effacés des désignants originaux, effacement qui donne lieu à des désignants synthétiques issus d'une condensation sémantique. Nous les appelons " motsévénements » (désormais ME) à la suite de Moirand. Ces expressions condensées « correspondent de fait à des opérations de référence à des événements, [et] fonctionnent comme des dénominations partagées 》 (2004 : 382). Comme résultat de la condensation d'une expression définie complète, le complément du nom se détache et devient apte à remplir une fonction référentielle. La conséquence de ce phénomène de condensation est que le caractérisant du Ndév est investi du contenu de toute l'expression définie. Si le Ndév apporte dans un premier temps une base lexicale fondamentale pour l'interprétation événementielle de l'expression, il cède ensuite sa force instructionnelle au noyau du complément, lequel se voit ainsi attribuer un rôle référentiel. Nous obtenons ainsi des toponymes événementiels (Hiroshima), des dates en fonction événementielle $\left(\right.$ Mai-68) ${ }^{8}$, des motsévénements « accidentels » (le voile) et des " phénonymes » (Katrina). Les anthroponymes ne peuvent pas remplir à long terme la fonction de désignant événementiel, de même que les noms de pays ${ }^{9}$, mais ils peuvent renvoyer temporairement à des événements, sans pour autant en constituer le nom-name.

\subsection{Les mots-événements}

Comme nous l'avons dit, le discours d'information procède toujours par catégorisation pour nommer un événement. Une dénomination comme Tiananmen n'est pas lancée telle quelle dans la presse, car elle ne serait pas capable de référer à un événement aux coordonnées spatio-temporelles définies; de la même façon, toute naturelle qu'elle nous semble maintenant, 1'expression le 11 septembre n'a pas toujours référé à un événement, et qui plus est, n'a pas toujours référé au même événement (mais au coup d'État

7. Pour une analyse détaillée de la formation des toponymes et des dates en fonction événementielle, voir Calabrese (2010b).

8. Pour les distinguer des noms de périodes ou chrononymes, nous les appelons héméronymes. Voir Calabrese 2008.

9. Même cas pour les noms de continents ou de régions assimilées à des pays :

- Europe : Nicolas Sarkozy tente d'imposer son idée de traité "simplifié" (lemonde.fr, 14 juin 2007)

- Gaza : le Hamas conquiert plusieurs positions du Fatah (lemonde.fr, 14 juin 2007) 
au Chili en 1973, par exemple). Mais une fois installés dans les discours publics, les ME ont une immense productivité sémiotique (notamment les toponymes et les héméronymes ou dates en fonction événementielle), car ils s'appuient sur un réseau sémantique épais ${ }^{10}$. Nous sommes tous capables de catégoriser, ne fût-ce que vaguement, les événements ainsi désignés, même en absence du Ndév original.

Pour comprendre leur fonctionnement il faut remonter aux expressions définies originales, car les DE s'appuient sur la mémoire lexicale du Ndév effacé, mais dont ils ont en quelque sorte "enregistré " l'information. Les expressions en après-événement sont la preuve que cette information lexicale continue à être présente :

(22) Après Tchernobyl, le monde s'interroge (La libre Belgique, 1-2 mai 1986)

(23) Vingt ans après Tiananmen, la censure continue (Metro, 4 juin 2009)

Ces exemples doivent être glosés après la catastrophe / l'accident de Tchernobyl et après le massacre de la place Tiananmen, car le Ndév confère une dimension durative au toponyme. Par ailleurs, le ou les Ndév gommés peuvent refaire surface en dehors du moment discursif correspondant. Lors des commémorations de l'accident de la centrale nucléaire de Tchernobyl, par exemple, les Ndév catastrophe et accident, qui avaient servi à constituer l'expression dénominative initiale, ont souvent été évoqués dans les articles de presse (Calabrese 2009).

La question que nous pouvons nous poser est comment s'actualise la mémoire de l'événement à partir d'un support aussi « simple », étant donné que les ME ne sont pas des dénominations à proprement parler? En effet, les $\mathrm{ME}$ fonctionnent comme des métonymies, dans le sens où la partie sert à identifier le tout. Dans un certain sens, on peut dire qu'ils ont enregistré la mémoire du Ndév qui a servi à les catégoriser. Les ME reposent ainsi sur une mémoire lexicale, mémoire interdiscursive s'il en est, car faisant référence à des énoncés antérieurs parus dans des numéros antérieurs du journal ${ }^{11}$. Si le constat semble banal, il est important de le rappeler, pour expliquer le fonctionnement mémoriel du discours de presse et l'incidence des routines rédactionnelles sur notre perception de l'actualité.

La formation des ME à partir de la mémoire lexicale d'une expression définie complète permet également d'expliquer les emplois métaphoriques comme les antonomases, très présentes dans le discours médiatique en général, de même que les périodisations :

10. Comme l'ont montré, dans le cas des toponymes, les travaux de Paveau (2008) et de Cislaru (2005).

11. Bien entendu, la perception de l'événement et les traces mémorielles qu'il laisse ne sont pas uniquement (ni avant tout) discursives, mais également visuelles. La mémoire de l'événement repose autant sur des mots (noms communs et noms propres, discours rapportés) que sur des images. 
(24) Genève, émue, s'interroge sur le " 11 septembre des Nations unies » (lemonde.fr 28.8.03)

(25) Vingt ans après Tiananmen, la censure continue (Metro, 4.6.09)

(26) De Hiroshima aux Twin Towers

(Le Monde diplomatique, $\mathrm{n}^{\circ}$ 582, septembre 2002)

Ces emplois sont la preuve de la mémoire discursive des ME, lesquels ne peuvent s'appliquer, dans les usages métaphoriques, qu'à des événements de la même nature. Or, il est important de situer cette mémoire : se trouve-t-elle dans le nom même? Dans le cerveau des lecteurs? Dans l'entourage textuel des désignants? Si la notion de mémoire discursive, reformulée en mémoire interdiscursive par Moirand, est un puissant outil d'analyse qui nous permet de repérer la présence d'énoncés antérieurs non marqués, faut-il l'entendre au sens littéral? Comme le demande Moirand, «ce seraient donc les mots qui ont une mémoire?» (2007: 51).

\section{La mémoire des désignants d'événements}

Si l'on se penche sur des désignants qui circulent massivement dans une société et sont pour cela largement partagés, on peut être tenté de penser qu'ils ont effectivement « enregistré » la mémoire des événements. Comme le note Moirand, il y aurait « comme quelque chose qu'on aurait déjà entendu [...] qui peut être de l'ordre du "vague", et qui serait stocké dans la mémoire du lecteur, sans quoi l'allusion ne peut être "sentie" et reste sans "effet" : mémorisation antérieure de formes, de sons, de sens, et même de constructions » (2007b: 6).

La question qui se pose est la suivante : en tant que lecteurs de la presse quotidienne, sommes-nous capables de décoder un désignant d'événement que nous ne connaissons pas, car nous n'identifions pas l'interdiscours dans lequel il s'inscrit? Autrement dit, sommes-nous capables d'identifier un désignant d'événement, notamment sous sa forme condensée de mot-événement, sans pour autant identifier son référent? Dans la grammaire du discours de presse, une série d'indices nous permettent de reconnaître qu'il s'agit d'un DE. Le titre bisegmental à deux points, par exemple, s'il ne se limite pas à la nomination d'événement, place souvent les désignants en position thématique, comme on le voit dans ces exemples :

(27) 11 mars : Zapatero met ETA hors de cause (nouvelobs.com, 18 décembre 2004) Le chef du gouvernement socialiste affirme que le « terrorisme international de type islamiste » est le seul responsable des attentats du 11 mars.

(28) Affaire Parmalat : onze responsables du groupe condamnés à des peines d'emprisonnement (lemonde.fr, 28 juin 05)

(29) Tsunami : les Indiens rendent hommage à leurs victimes un an après (lemonde.fr, 25 décembre 2005) 
En particulier, les toponymes événementiels et les dates en fonction événementielle incitent à chercher le Ndév effacé en mémoire stable, puisqu'ils contiennent une des coordonnées principales de l'événement (le lieu ou la date). Prenons l'exemple d'un toponyme événementiel qui désigne un événement lié à l'histoire contemporaine de la France :

(30) Tibéhirine et après (lemonde.fr, 21 novembre 2009)

Sans les prédiscours nécessaires, quels sont les indices d'événementialité du toponyme? La construction syntaxique « nom propre + après » déclenche l'instruction de chercher un référent en mémoire stable : qu'est-ce que Tibéhirine / que s'est-il passé à Tibéhirine ? L'information nécessaire est dans le cotexte :

(30) Connaîtra-t-on un jour la vérité sur l'assassinat des moines de Tibéhirine, il y a plus de treize ans, en Algérie? L'affaire, trouble à souhait, nimbée de raison d'État, pourrait enfin s'éclaircir si les autorités françaises levaient tous les obstacles auxquels la justice se heurte depuis cinq ans.

Ainsi, il est toujours possible de récupérer les interdiscours manquants et d'identifier les Ndév effacés. Pour un lecteur étranger à l'actualité belge, par exemple, le titre suivant peut paraître obscur :

(31) Un Ghislenghien bis reste encore possible (Metro, 27 juillet 2006)

Or, une recherche sur Google montre à quel point les Ndév effacés du mot-événement sont présents dans les discours sociaux, car nous retrouvons les noms communs catastrophe, explosion et accident qui ont participé de la construction de l'événement. Même si ces informations ne sont pas hiérarchisées, et sont décontextualisées et privées de leurs sources, la circulation de ces Ndév associés au toponyme témoigne du partage des savoirs liés à l'événement, savoir qui repose sur une base lexicale proposée par le discours d'information.

Reposons une fois encore la question à la lumière des observations ici avancées : où est située la mémoire? Le sens est-il contenu dans le nom? Comment devons-nous envisager le rapport entre la nomination d'événements et la mémoire : du point de vue de nos souvenirs - nous nous souvenons de l'événement - ou bien du point de vue de l'information contenue dans le désignant - le désignant porte la mémoire de l'événement? Si nous n'avons pas assisté au moment discursif correspondant et que nous ne sommes pas en possession des prédiscours adéquats, la référenciation échoue. Cela prouve que le désignant ne porte pas en soi plus d'instructions sémantiques que celles qui appartiennent au contenu notionnel ou instructionnel : par exemple, la canicule ou le tsunami comportent l'instruction de chercher un référent événementiel, mais ne nous disent rien sur les coordonnées de l'événement. Même cas pour les DE formés à partir du suffixe-gate, qui s'inscrivent dans la lignée des scandales politiques par 
analogie au scandale du Watergate. Au contraire, dans Ghislenghien ou le 11 mars, nous connaissons une coordonnée de l'événement (le lieu ou la date), mais pas sa nature. C'est donc le sujet qui est porteur de mémoire, et non le mot, mais c'est bien ce dernier qui fonctionne comme un déclencheur mémoriel, comme le dit Moirand. Dans ce sens, les ME font partie de ce que Marie-Anne Paveau appelle la « technologie discursive » qui rend possible le partage d'amples domaines de connaissance collective :

Il peut s'agir d'outils linguistiques (grammaires, dictionnaires, mémentos, listes, guides de conversation, essais puristes, etc.), d'écrits et d'inscriptions de toutes sortes [...], et de nombreux autres artefacts comme les blocs-notes, les listes, les carnets d'adresse, les agendas, les calendriers, etc. Il peut s'agir aussi, comme le signale B. Conein, de « données publiques, comme tous les artefacts permettant d'acquérir, de stocker, de visualiser une quantité d'informations qu'un seul individu ne peut traiter ». (2006:132)

On peut donc considérer les désignants d'événements comme des outils cognitifs, dans le sens où ils permettent d'accéder à une mémoire qui, située chez l'individu, est construite collectivement dans les discours publics. Le modèle de la cognition distribuée, dans lequel s'inscrit la théorisation de Paveau, permet de situer la mémoire à la fois chez l'individu et dans le mot. La mémoire discursive est ainsi « délocalisée » du sujet, car le contenu événementiel d'un nom ne peut être actualisé, déclenché qu'en fonction des prédiscours dont le sujet dispose.

Ainsi posée, la problématique est à la fois linguistique (car il faut tenir compte de la base lexicale des expressions dénominatives), sémiotique (car il faut considérer les indices matériels qui suggèrent la présence d'un DE), discursive (puisque le fonctionnement des DE dépend des règles propres au discours d'information) et cognitive (dans la mesure où il faut un sujet capable d'actualiser la mémoire interdiscursive).

\section{Conclusion}

Nous avons essayé de montrer les mécanismes par lesquels le discours d'information médiatique construit la mémoire de l'actualité par le biais des désignants d'événements. Si tous les DE constituent, dans le cadre $\mathrm{du}$ discours d'information, des dispositifs de mémorisation de l'histoire immédiate, car ils sont capables d'évoquer une grande quantité d'information, ils n'ont pas tous les mêmes capacités mémorielles. C'est là que la description linguistique intervient pour explorer les différentes formes d'évocation du toponyme, des dates, des noms communs, des expressions définies complètes ou incomplètes. Si les toponymes et les dates sont des contenants privilégiés de la mémoire historico-médiatique, c'est parce que seule une convention dénominative stable et à long terme - comme celle du nom propre - peut garantir la référenciation, ce qui expliquerait ces cas emblématiques que sont Mai 68, le 11 septembre ou 
Tchernobyl. Ces mots-événements très saillants s'insèrent dans les temps longs de l'événement historico-médiatique, c'est-à-dire qu'ils circulent en dehors du moment discursif. Au contraire, les expressions dénominatives incomplètes, trop dépendantes à la fois de leur sens lexical et du contexte d'énonciation, ont une capacité mémorielle plus faible. Un cas fait pourtant exception : les xénismes, qui comptent parmi les expressions dénominatives incomplètes (l'intifada ou le tsunami), se rapprochent plus du nom propre que du nom commun, en raison de leur opacité sémantique, et ont une capacité d'évocation plus grande qu'un substantif. Dans le cas précis des mots-événements, il s'agissait de montrer comment leur mémoire interdiscursive reposait sur l'effacement d'un nom événementiel, lequel continuait à circuler dans les discours publics.

Une fois les mots-événements installés, ils jouent un rôle central dans notre perception de l'actualité. Non seulement ils participent de la construction de l'histoire immédiate, mais également de la conservation de la mémoire du passé, notamment lors de commémorations, d'anniversaires ou simplement d'antonomases et de périodisations. Dans leur rôle de déclencheurs mémoriels, ils contribuent à organiser le temps public, qu'ils scandent en un avant et un après ou qu'ils divisent en périodes (l'aprèsKyoto, l'après-Tchernobyl), et ils organisent l'expérience publique, en créant des familles d'événements par le biais de l'antonomase (L'Amérique sous le choc d'un "Pearl Harbor » terroriste ${ }^{12}$ ) et en tissant des liens de causalité (De Hiroshima aux Twin Towers ${ }^{13}$ ).

Laura Calabrese Steimberg

Université Libre de Bruxelles - Ladisco

\section{Références}

Badiou, A., 1988, L'Être et l'Événement, Paris, Seuil.

Calabrese, L., 2008, « Les héméronymes : ces événements qui font date, ces dates qui deviennent événements », Mots. Les Langages du politique, $\mathrm{n}^{\circ} 88$, p. $115-128$.

—, 2009, « Nom propre et dénomination événementielle : quelles différences en langue et en discours? "), Corela, vol. 7, n 1, http://edel.univpoitiers.fr/corela/document.php $\mathrm{id}=2119$.

12. Le Monde, 13 septembre 2001.

13. Le Monde diplomatique, septembre 2002. 
—, 2010a, Le rôle des désignants d'événements dans la construction de l'histoire immédiate. Une analyse du discours de la presse écrite, Thèse de doctorat, Bruxelles, Université Libre de Bruxelles.

—, 2010b, «Le réemploi de dénominations d'évènements dans la construction d'évènements prototypiques ", dans le IVe colloque international Ci-Dit, http://revel.unice.fr/symposia/cidit/index.html ?id=398.

Charolles, M., 2002, La référence et les expressions référentielles en français, Paris, Ophrys.

Cislaru, G., 2005, Étude sémantique et discursive du nom de pays dans la presse française avec référence à l'anglais, au roumain et au russe, Thèse en sciences du langage, Paris, Université Paris 3 Sorbonne nouvelle.

Fabre, C. et Le Draoulec, A., 2006, « La dimension événementielle du syntagme nominal dans la structure avant $+\mathrm{SN} »$, Cahiers de Lexicologie, $\mathrm{n}^{\circ} 89$, p. 47-74.

Kaufmann, L., 2008, " La société de déférence. Médias, médiations et communication », Réseaux, vol. 26, nº 148-149, p. 79-116.

Lecolle, M., 2009, « Changement de sens du toponyme en discours : de Outreau "ville" à Outreau "fiasco judiciaire" ", dans M. Lecolle, M.A. Paveau et S. Reboul-Touré (dir.), Les Carnets du Cediscor, $\mathrm{n}^{\circ} 11:$ Le Nom propre en discours, p. 91-106.

—, 2004, « La circulation interdiscursive comme lieu de construction de domaines de mémoire par les médias », dans J.-M. López Muñoz, S. Marnette et L. Rosier (dir.), Le Discours rapporté dans tous ses états, Paris, L'Harmattan, p. 373-385.

Moirand, S., 2007, Observer la presse quotidienne, Paris, PUF.

Paveau, M.-A., 2006, Les Prédiscours. Sens, mémoire, cognition, Paris, Presses Sorbonne Nouvelle.

—, 2008, « Le toponyme, désignateur souple et organisateur mémoriel. L'exemple du nom de bataille », dans H. Boyer et M.-A. Paveau (dir.), Mots. Les langages du politique, $\mathrm{n}^{\circ} 86$ : Toponymes. Instruments et enjeux, p. 23-35.

Quéré, L., 1994, "L'événement "sous une description" : contraintes sémantiques, croyances stéréotypiques et "natural facts of life as a morality'», Protée, no 22 (2), p. 14-28.

Searle, J., 1998 [1995], La Construction de la réalité sociale (trad. française de C. Tiercelin), Paris, Gallimard.

Veniard, M., 2007, La nomination d'un événement dans la presse quotidienne nationale. Une étude sémantique et discursive : la guerre en Afghanistan et le conflit des intermittents dans Le Monde et Le Figaro, Thèse de doctorat, Université Paris 3 Sorbonne nouvelle.

Veyne, P., 1996 [1971], Comment on écrit l'histoire, Paris, Seuil. 